\title{
Parity-violating Kalb-Ramond-Maxwell interactions and CMB anisotropy in a braneworld
}

\author{
Debaprasad Maity ${ }^{* a}$, Parthasarathi Majumdar ${ }^{\dagger b}$ and Soumitra SenGupta ${ }^{\ddagger a}$ \\ a Department of Theoretical Physics, Indian Association for the Cultivation of Science, Kolkata 700 032, India \\ ${ }^{b}$ Theory Group, Saha Institute of Nuclear Physics, AF/1 Bidhannagar, Kolkata 700 064, India
}

\begin{abstract}
Following up on a recent paper by two of us (DM and SS), demonstrating the large enhancement in observable optical activity in radiation from high redshift sources arising from the stringbased coupling of bulk Kalb-Ramond field to the Maxwell Chern Simons three-form on the brane in a Randall-Sundrum braneworld, we exhibit here a similar enhancement in parity-violating temperature-polarization correlations, yet unseen, in the CMB anisotropy due to a generalized parity-violating Kalb-Ramond axion-photon interaction proposed earlier by one of us (PM). The non-observation of such correlations in CMB anisotropies would necessitate unnatural fine tuning of the Kalb-Ramond axion parameters. As a stringy realization of Randall-Sundrum braneworld scenario is yet to be understood properly, our work indicates the need of a careful investigation to establish the connection between string-based phenomenological models and the Randall-Sundrum braneworld scenario.
\end{abstract}

The ubiquity of the Kalb-Ramond (KR) antisymmetric tensor field in massless spectra of all closed string theories makes it, along with the dilaton, a special low energy degree of freedom naturally occurring in a stringy world. On the other hand, the standard strong-electroweak theory does not appear at all to need such a field for an adequate description of $\mathrm{TeV}$ scale phenomena. Therefore any observational evidence at low energies of KR fields would point to physics beyond the standard strong-electroweak theory. It stands to reason that such an evidence would be indicative of an underlying fundamental theory whose tree level spectrum must resemble closely that of some sort of the string (M-) theory. The focus of this paper is precisely on such possible indirect observational evidence of KR fields.

The KR field is known in heterotic string theory [1] to have a coupling, induced at the quantum level, to the YangMills Chern-Simons three-form. Basically, it entails augmenting the KR three-form field strength $H \rightarrow H+\Omega_{Y}$ where $\Omega_{Y}$ is the Yang Mills Chern Simons three-form. This coupling originates from the requirement of quantum consistency (cancellation of anomalies) and $N=1$ supersymmetry of the theory. The interaction survives compactification to four dimensions, leading to the low energy effective interaction [2] of the form $S_{\text {int }} \sim\left(1 / M_{P}\right) \int d^{4} x \phi_{H} F{ }^{*} F$, where $\phi_{H}$ is the pseudoscalar field (KR axion) dual to the KR field strength, $F$ is the Maxwell field strength and $M_{P}$ is the Planck mass. Assuming a homogeneous background KR field with little backreaction on electromagnetic fields, this coupling has been shown [3], [4] to lead to an optical activity in electromagnetic radiation from high redshift sources in the form of rotation of the plane of polarization by an angle $\Delta \Phi=h t(z)$. Here $h$ is essentially $d \phi_{H} / d t$, and $t(z)$ is the 'lookback' time as a function of the redshift $z$. Observational upper limits on $\Delta \Phi[5]$ bound $h$ from above by a number of $O\left(10^{-5}\right)$ in units of Planck mass.

A different possibility seems to appear if the same interaction is considered in a higher dimensional low energy effective model proposed about five years ago - the Randall-Sundrum (RS) scenario [6]. Although so far this model has not been derived in detail from string (M-) theory, compactifications with non-vanishing fluxes of $p$-form gauge fields in the Ramond-Ramond sector appear to generate scenarios similar to the RS model $[7,8]$. Furthermore, there is as yet no indication that the RS model contradicts any basic tenet of M-theory compactifications.

In the RS scenario, massless closed string modes like the KR field, (alongwith gravitons) propagate in the bulk (i.e., in all spacetime dimensions including the compact one) while all the standard model fields (open string modes) are confined to the brane. Of course, there are interactions between closed and open string modes, as required by string consistency. In this paper we shall concentrate on one such particular interaction, namely the interaction between the KR field (in the bulk) and the Maxwell field on the visible brane. In this context, the heterotic string has a special significance since its spectrum in principle accommodates all fields of the standard model, including the Maxwell field (after appropriate compactification). KR-Maxwell couplings occurring in the heterotic string are therefore of particular relevance here. As already mentioned above, the KR field strength tensor couples to the Chern Simons

\footnotetext{
*E-mail: tpdm@iacs.res.in

${ }^{\dagger}$ On deputation from the Institute of Mathematical Sciences, Chennai 600 113, India; E-mail: partha@theory.saha.ernet.in

${ }^{\ddagger}$ E-mail: tpssg@iacs.ernet.in
} 
three form of the Maxwell field in the heterotic string.

Recently, two of the authors [9] have considered such an interaction of the Maxwell Chern Simons three-form to a homogeneous KR background within the RS braneworld scenario. The analysis shows that the coupling is enhanced by the inverse of the warp factor in the five dimensional RS metric. We may recall that this warp factor accounts for the exponential suppression which ensures naturalness of the electroweak theory by making the Higgs mass stay close to the weak scale; it is a number of $O\left(10^{-16}\right)$. With the inverse of this factor appearing in the KR-Maxwell coupling, the optical rotation of radiation from large redshift sources increases enormously. To reconcile the resultant theoretical angle of rotation with the (non-)observations, one needs to fine tune the parameter $h$ (in units of the Planck scale) to a precision of less than one part in $10^{15}$ - certainly unnatural from the standard 'naturalness' perspective.

In this paper, we extend the analysis of [9], so as to be applicable to the anisotropy of the Cosmic Microwave Background ( $\mathrm{CMB}$ ) through the introduction of spatial parity $(\mathrm{P}-)$ violation. It has been noted several years ago [10] that certain non-vanishing multipole moment correlations between the temperature anisotropy and polarization of the CMB could appear, if in the interaction described above, the KR axion was to be replaced by a homogeneous scalar field, leading to P-violation. Such an interaction appears in a proposal in [11] in the form of an extended augmentation, of the odd parity KR field strength, by a term which has even parity. As we shall explain below, this can produce $\mathrm{P}$-violating multiple moment correlations of temperature anisotropy and polarization of the CMB, without having to change parity of the free KR field. With this additional augmentation of the KR field strength, the analysis of [9] now carries over easily to the CMB case, as we proceed to describe.

The angular distribution of the temperature anisotropy of the CMB has the well-known expansion in spherical harmonics [13]

$$
\frac{\Delta T}{T}(\mathbf{n})=\sum_{l, m} a_{l m}^{T} Y_{l m}^{T}(\mathbf{n})
$$

The polarization of the CMB is expressed in terms of a $2 X 2$ traceless symmetric tensor $\mathcal{P}_{a b}(\mathbf{n})$ whose components are the Stokes parameters. This tensor can be decomposed into its irreducible 'gradient' (or $E$ ) and 'curl' (or $B$ ) parts which have opposite spatial parity. The angular distribution of this polarization tensor can thus be expressed in terms of matrix spherical harmonics as [10]

$$
\begin{aligned}
& \mathcal{P}_{a b}^{E}(\mathbf{n})=\sum a_{l m}^{E} Y_{l m, a b}^{E}(\mathbf{n}) \\
& \mathcal{P}_{a b}^{B}(\mathbf{n})=\sum a_{l m}^{E} Y_{l m, a b}^{B}(\mathbf{n}) .
\end{aligned}
$$

One defines correlations of the multipole moment coefficients $a_{l m}^{X}, X=T, E, B$ as

$$
C_{l}^{X X^{\prime}} \equiv\left\langle a_{l m}^{X} a_{l m}^{X^{\prime}}\right\rangle
$$

Clearly, correlations such as $C_{l}^{X X}$ all preserve $\mathrm{P}$, as do correlations like $C_{l}^{T E}$, while correlations like $C_{l}^{T B}$ or $C_{l}^{E B}$ are obviously $\mathrm{P}$-violating, requiring an explicitly $\mathrm{P}$-violating interaction as mentioned earlier. The optical activity described earlier implies that if a correlation like $C_{l}^{T E}$ does indeed arise due to reionization or otherwise, the passage of the Thompson scattered photons through the KR background will produce the P-violating correlations $C_{l}^{T B}$ through the rotation $[10]$

$$
C_{l}^{T B}=C_{l}^{T E} \sin 2 \Delta \Phi .
$$

Such a parity violating effect has been shown to arise from a phenomenological action of the type [10]

$$
S_{i n t} \sim \int \phi F \cdot^{*} F
$$

where $\phi$ is an even parity scalar.

Let us now see how such P-violation may possibly arise within a string theoretic framework. The standard augmentation of the KR field strength is given, at the effective $4 \mathrm{~d}$ field theory stage, by

$$
H_{\mu \nu \lambda} \rightarrow \tilde{H}_{\mu \nu \lambda} \equiv H_{\mu \nu \lambda}+\frac{1}{3 M_{P}} A_{[\mu} F_{\nu \lambda]} .
$$

We now propose, following [11], the extended version of augmentation of the KR field strength 


$$
\tilde{H}_{\mu \nu \lambda}=H_{\mu \nu \lambda}+\frac{1}{3 M_{P}}\left[\zeta_{+} A_{[\mu} F_{\nu \lambda]}+\zeta_{-} A_{[\mu}{ }^{*} F_{\nu \lambda]}\right],
$$

where, $\zeta_{ \pm}$are $O(1)$ dimensionless real numbers. The first term is the standard Chern Simons augmentation, while the second is our extension; its main purpose is to contaminate the odd parity KR field strength with a small (due to Planck mass suppression) even parity impurity. Since $\tilde{H}^{2}$ appears in the effective action, it must be invariant under both the KR and Maxwell gauge transformations. The latter invariance requires that the unaugmented $H_{\mu \nu \lambda}$ be assigned a Maxwell $U(1)$ gauge transformation

$$
\delta_{U} H_{\mu \nu \lambda}=-\frac{1}{3 M_{P}} \partial_{[\mu} \omega\left[\zeta_{+} F_{\nu \lambda]}+\zeta_{-}^{*} F_{\nu \lambda]}\right] .
$$

An immediate consequence of this gauge transformation is that the $H_{\mu \nu \lambda}$ now can no longer be thought of as a parity eigenstate, and therefore neither can its dual spinless field $\phi_{H}$. In other words, one can decompose

$$
\phi_{H}=\phi_{H}^{(+)}+\phi_{H}^{(-)}
$$

where $\phi_{H}^{( \pm)}$has parity \pm . It is now easy to show [11] that the interaction part of the effective action contains a P-violating term

$$
S_{\text {int }}^{\mathrm{PV}} \sim M_{P}^{-1} \int \phi_{H}^{(+)} F *^{*} F,
$$

i.e., precisely the sort of coupling that leads to the P-violating correlations $C_{l}^{T B}$ in the CMB anisotropy. We note in passing that the $\mathrm{P}$-violating interaction also violates time reversal invariance ( $\mathrm{T}$ ) so as to leave $\mathrm{PT}$ invariant [10].

A few remarks about the extended augmentation are in order: first of all, unlike the Chern Simons augmentation, it is not topological (nonlocal) in nature and hence does not introduce any new anomalies. It is most likely a local counterterm at the quantum level. Such counterterms commonly occur in local field theories so long as they obey all gauge invariances. Their determination requires inputs ('renormalization conditions') related to experiment.

Second, it can easily be embedded in a supersymmetric theory at the $N=1, D=4$ level. To see this, recall that the locally supersymmetric Maxwell action in $N=1, D=4$ superspace has the form

$$
S_{M a x} \sim \int d^{4} x d^{2} \theta \chi(\mathcal{S}) W^{\alpha} W_{\alpha}+\text { h.c. },
$$

where the gauge kinetic function $\chi(\mathcal{S})$ is an analytic function of the chiral superfield $\mathcal{S}$ whose top component is the complex scalar $\phi_{D}+i \phi_{H}$. If this gauge kinetic function $\chi(\mathcal{S}) \rightarrow \zeta_{+} \chi(\mathcal{S})+\zeta_{-} \tilde{\chi}(i \mathcal{S})$, the component action indeed has the interaction terms discussed above. With such features one expects the extension proposed above to be consistent with quantum string theory.

As argued in [11], the calculation of the angle of rotation of the polarization plane of the radiation is very similar to that in [3]. There are however additional physical effects of this extended augmentation. The most important of these is the possibility of an effective time dependent fine structure constant occurring because of the P-conserving interaction

$$
S_{i n t}^{P}=\frac{\zeta_{-}}{M_{P}} \int d^{4} x \phi_{H}^{(+)} F^{2}
$$

For a homogeneous $\phi_{H}^{(+)}=\phi_{H}^{(+)}(t)$, the (12) can be added on to the Maxwell action leading to an effective $\alpha_{e f f}(t)$ given by

$$
\alpha_{e f f}^{-1}(t)=\alpha_{0}^{-1}+\frac{\zeta_{-}}{M_{P}} \phi_{H}(t)
$$

Given that $\phi_{H}^{(+)}(t)=\zeta_{-}\left(h / M_{P}\right) t(z)$, where $t(z)$ is the lookback time, eq. (13) implies that the effective fine structure constant was smaller in the past. Using upper limits obtained recently on the time variation of the fine structure constant [14], one may bound the parameter $\zeta_{-} h$ from above. This implies that the appearance of P-violating multipole moments in the CMB anisotropy will be correlated with the time-variation of the effective fine structure constant, if, as per our proposal here, they both originate from the same P-violating interaction of the KR field.

One now comes to the interesting question as to what happens when the P-violating interaction described above is superimposed on the warp factor-enhanced KR-Maxwell Chern Simons coupling in the RS scenario obtained in [9]. We work, following [9], within a five dimensional RS scenario described by the metric 


$$
d s^{2}=e^{-2 \sigma} \eta_{\alpha \beta} d x^{\alpha} d x^{\beta}+r_{c}^{2} d \phi^{2}
$$

where $\sigma=k r_{c}|\phi|$. The augmentation of the KR field, propagating in the bulk, is given by the Maxwell Chern Simons three-tensor on the brane together with the extension proposed above

$$
\tilde{H}_{M N L}=\partial_{[M} B_{N L]}+\frac{1}{3 M_{p}^{1 / 2}} \delta_{M}^{\mu} \delta_{N}^{\nu} \delta_{L}^{\lambda} A_{[\mu}\left[\zeta_{+} F_{\nu \lambda]}+\zeta_{-}^{*} F_{\nu \lambda]}\right] .
$$

This augmentation leads to the interaction

$$
\begin{gathered}
S_{\text {int }}=\frac{1}{3 M_{p}^{\frac{1}{2}}} \int d^{5} x \sqrt{-g} \delta_{\mu}^{M} \delta_{\nu}^{N} \delta_{\lambda}^{L} \delta(\phi-\pi) H_{M N L} \\
A^{[\mu}\left[\zeta_{+} F^{\nu \lambda]}+\zeta_{-}{ }^{*} F^{\nu \lambda]}\right] .
\end{gathered}
$$

Now, the KR potential $B_{\mu \nu}(x, \phi)$ in the bulk has the Kaluza-Klein decomposition

$$
B_{\mu \nu}(x, \phi)=\sum_{n=0}^{\infty} B_{\mu \nu}^{n}(x) \chi^{n}(\phi) \frac{1}{\sqrt{r_{c}}}
$$

The $4 \mathrm{~d}$ effective action for $B_{\mu \nu}$ on the brane is expected to have the form

$$
S_{H}=\int d^{4} x \sum_{n=0}^{\infty}\left[\eta^{\mu \alpha} \eta^{\nu \beta} \eta^{\lambda \gamma} H_{\mu \nu \lambda}^{n} H_{\alpha \beta \gamma}^{n}+3 m_{n}^{2} \eta^{\mu \alpha} \eta^{\nu \beta} B_{\mu \nu}^{n} B_{\alpha \beta}^{n}\right]
$$

where the modes $\chi^{n}$ satisfy

$$
-\frac{1}{r_{c}^{2}} \frac{\partial^{2} \chi^{n}}{\partial \phi^{2}}=m_{n}^{2} \chi^{n} e^{2 \sigma}
$$

Following [12], we infer the existence of a constant zero mode

$$
\chi^{0}=\sqrt{k r_{c}} e^{-k r_{c} \pi}
$$

Using eq. (16), one obtains the interaction

$$
\begin{gathered}
S_{i n t}=\frac{r_{c}}{M_{p}^{\frac{1}{2}}} \int d^{4} x d \phi e^{2 \sigma} \delta(\phi-\pi) \eta^{\mu \rho} \eta^{\nu \sigma} \eta^{\lambda \xi} H_{\mu \nu \lambda}(x, \phi) \\
\mathrm{X} A_{[\rho}\left[\zeta_{+} F_{\sigma \xi]}+\zeta_{-}{ }^{*} F_{\sigma \xi]}\right] .
\end{gathered}
$$

Observe that the contraction of the KR field strength with the Maxwell three-tensor fields has entailed the use of several inverse four-metric components which has resulted in not only cancelling all the warp factors, but also in an extra inverse warp factor leftover in the integrand in (21). Integrating over the bulk coordinate $\phi$ and using eq. (20) one obtains the effective interaction on the brane

$$
S_{i n t}=\sqrt{\frac{k}{M_{p}}} r_{c} e^{k r_{c} \pi} \int d^{4} x H_{\mu \nu \lambda}(x) A^{[\mu}\left[\zeta_{+} F^{\nu \lambda]}+\zeta_{-}{ }^{*} F^{\nu \lambda]}\right] .
$$

Replacing the KR field strength by its dual $\phi_{H}$ and using the decomposition (9) into parity eigenstates, one obtains the braneworld version of eq. (10)

$$
S_{i n t}^{\mathrm{PV}}=\sqrt{\frac{k r_{c}^{2}}{M_{P}}} \exp \left\{\pi k r_{c}\right\} \zeta_{+} \int \phi_{H}^{(+)} F \cdot^{*} F
$$

Just as in [9], the optical rotation due to this interaction will be huge, in view of the exponential enhancement factor $\exp \left\{\pi k r_{c}\right\}$, and from eq. (23) therefore one expects large P-violating multipole moment correlations from eq. (4) 
in the CMB anisotropy. Once again, the only way to control this runaway enhancement will be to fine tune $h$ to unnaturally small values ${ }^{1}$.

The situation is equally compelling for the time variation of the fine structure constant; to obtain the observed relative variation of $O\left(10^{-5}\right)$ per year [14], unnatural fine tuning of $\zeta_{-} h$ seems imperative. We have assumed that $\zeta_{-}=O(1)$ so that reconciling with observations amounts to unnatural fine tuning of $h$.

To conclude, starting from an extended augmentation of the KR field strength beyond the string theory motivated Chern Simons augmentation, and having a different spatial parity from the latter, and using a five dimensional RS framework as our setting, we have demonstrated the possibility of occurrence of abnormally large P-violating temperature-polarization multipole moment correlations in the CMB anisotropy. The model also predicts abnormally large time variations of the fine structure constant. Both these require fine tuning of the KR parameter $h / M_{P}$ to unnaturally small values in order to be reconciled with (non-)observations.

In conclusion this work shows a possible difficulty one may encounter to establish a connection between stringy KR background and Randall-Sundrum braneworld. However keeping in mind that a proper stringy realization of RandallSundrum braneworld model is yet to be formulated, our work may play a crucial role in finding this connection.

One of us (SS) thanks M. Kamionkowski for stimulating correspondence related to possible generalization of the work in [9] to the case of CMB anisotropy. Another of us (PM) thanks D. Bailin, F. Barbero, S. Bharadwaj, A. Chatterjee, T. Souradeep, S. Theisen and S. Umasankar for discussions, and the Theory Group at CERN, the Theoretical Physics Group at IMAFF, the Department of Physics at the University of Sussex and the Albert Einstein Institute for hospitality during the various stages of completion of this work. DM acknowledges the Council for Scientific and Industrial Research, Government of India for financial support.

[1] M. Green, J. Schwarz and E. Witten 1987, Superstring Theory, vol. 2, p. 382 et.seq. (Cambridge)

[2] P. Majumdar and S. Sengupta 1999, Class. Quant. Grav. 16 L89.

[3] S. Kar, P. Majumdar, S. SenGupta and A. Sinha 2002, Eur. Phys. Jour. C23 357.

[4] S. Kar, P. Majumdar, S. SenGupta and S. Sur 2002, Class. Quant. Grav. 19677.

[5] J. Wardle, R. Perley and M. Cohen 1997, Phys. Rev. Lett. 791801.

[6] L. Randall and R. Sundrum 1999, Phys. Rev. Lett. 83, 3370; ibid. 83, 4690.

[7] S.B. Giddings, S. Kachru and J. Polchinski 2002, Phys. Rev D66 106006 ; hep-th/0105097.

[8] C. P. Herzog, I.R. Klebanov, P. Ouyang, arXiv:hep-th/0205100 and references therein.

[9] D. Maity and S. SenGupta 2003, Cosmic optical activity in a Randall-Sundrum Braneworld with torsion, arXiv:hepth/0311142.

[10] A. Lue, L. Wang and M. Kamionkowski 1999, Phys. Rev. Lett. 831506 ; N. Lepora, gr-qc/9812077.

[11] P. Majumdar 2001, New parity-violating photon-axion interactions, arXiv:hep-th/0105122; see also, B. Mukhopadhyay, S. SenGupta and S. Sur 2001, Mod. Phys. Lett. A17 43.

[12] B.Mukhopadhyaya, S.Sen, S.SenGupta 2002, Phys. Rev. Lett.89 121101.

[13] M. White and J. Cohn 2002, TACMB-1, arXiv:astro-ph/0203120.

[14] J. Webb et. al. 2001, Phys. Rev. Lett. 87091301.

\footnotetext{
${ }^{1}$ First numerical estimates on how precisely one must fine tune $h / M_{P}$ are in the $O\left(10_{15}\right)$ range; this will be elaborated elsewhere.
} 